\title{
Nonparametric Interrogation of Transcriptional Regulation in Single-Cell RNA and Chromatin Accessibility Multiomic Data
}

\author{
Yuriko Harigaya \\ University of North Carolina \\ Zhaojun Zhang \\ University of Pennsylvania \\ Hongpan Zhang \\ University of Virginia \\ Chongzhi Zang \\ University of Virginia \\ Nancy Zhang ( $\nabla$ nzh@wharton.upenn.edu ) \\ University of Pennsylvania https://orcid.org/0000-0002-0880-5749 \\ Yuchao Jiang \\ University of North Carolina
}

Article

Keywords: single-cell multiomics, transcriptional regulation, transcription factor, chromatin accessibility

Posted Date: September 28th, 2021

DOI: https://doi.org/10.21203/rs.3.rs-930184/v1

License: (c) (i) This work is licensed under a Creative Commons Attribution 4.0 International License.

Read Full License 


\section{Nonparametric Interrogation of Transcriptional Regulation in Single-Cell RNA and Chromatin Accessibility Multiomic Data}

Yuriko Harigaya ${ }^{1}$, Zhaojun Zhang ${ }^{2}$, Hongpan Zhang ${ }^{3,4}$, Chongzhi Zang ${ }^{3,4,5}$, Nancy R Zhang ${ }^{2, *}$, Yuchao Jiang $6,7,8,{ }^{*}$

1 Curriculum in Bioinformatics and Computational Biology, School of Medicine, University of North Carolina, Chapel Hill, NC 27599, USA.

2 Department of Statistics, The Wharton School, University of Pennsylvania, Philadelphia, PA 19104, USA.

3 Center for Public Health Genomics, University of Virginia, Charlottesville, VA 22908, USA.

4 Department of Biochemistry and Molecular Genetics, University of Virginia, Charlottesville, VA 22908, USA.

5 Department of Public Health Sciences, University of Virginia, Charlottesville, VA 22908, USA.

6 Lineberger Comprehensive Cancer Center, University of North Carolina at Chapel Hill, NC 27599, USA.

7 Department of Genetics, School of Medicine, University of North Carolina, Chapel Hill, NC 27599, USA.

8 Department of Biostatistics, Gillings School of Global Public Health, University of North Carolina, Chapel Hill, NC 27599, USA.

* To whom correspondence should be addressed: nzh@wharton.upenn.edu, yuchaoj@email.unc.edu. 


\section{Abstract}

2 Epigenetic control of gene expression is highly cell-type- and context-specific. Yet, 3 despite its complexity, gene regulatory logic can be broken down into modular

4 components consisting of a transcription factor (TF) activating or repressing the 5 expression of a target gene through its binding to a cis-regulatory region. Recent 6 advances in joint profiling of transcription and chromatin accessibility with single-cell 7 resolution offer unprecedented opportunities to interrogate such regulatory logic. Here, 8 we propose a nonparametric approach, TRIPOD, to detect and characterize three-way 9 relationships between a TF, its target gene, and the accessibility of the TF's binding site, 10 using single-cell RNA and ATAC multiomic data. We apply TRIPOD to interrogate cell11 type-specific regulatory logic in peripheral blood mononuclear cells and contrast our 12 results to detections from enhancer databases, cis-eQTL studies, ChIP-seq experiments, 13 and TF knockdown/knockout studies. We then apply TRIPOD to mouse embryonic brain 14 data during neurogenesis and gliogenesis and identified known and novel putative 15 regulatory relationships, validated by ChIP-seq and PLAC-seq. Finally, we demonstrate

16 TRIPOD on SHARE-seq data of differentiating mouse hair follicle cells and identify 17 lineage-specific regulation supported by histone marks for gene activation and super18 enhancer annotations.

20 Keywords: single-cell multiomics, transcriptional regulation, transcription factor, 21 chromatin accessibility. 
22 Context-specific regulation of gene transcription is central to cell identity and function in 23 eukaryotes. Precision of transcriptional control is achieved through multitudes of 24 transcription factors (TFs) that bind to the cis-regulatory regions of their target genes, 25 dynamically modulating chromatin accessibility and recruiting transcription complexes in 26 response to developmental and environmental cues ${ }^{1}$. Dissecting this regulatory logic is 27 fundamental to our understanding of biological systems and our study of diseases. Over 28 the past decades, molecular studies have elucidated the structure of TF complexes and 29 provided mechanistic models into their function ${ }^{2}$. Methods based on high-throughput sequencing have enabled the genome-wide profiling of gene expression ${ }^{3}$, TF binding ${ }^{4}$, 31 chromatin accessibility ${ }^{5}$, and 3D genome structure ${ }^{6}$. TF knockdown/knockout studies 32 have also identified, en masse, their species-, tissue-, and context-specific target genes ${ }^{7}$. 33 Concurrently, novel statistical approaches have allowed for more precise identification 34 and modeling of TF binding sites $^{8}$, and expression quantitative trait loci (eQTLs) 35 databases now include associations that are tissue-specific ${ }^{9}$ and will soon be cell-type specific ${ }^{10}$. Yet, despite this tremendous progress, our understanding of gene regulatory logic is still rudimentary.

When a TF $j$ activates or represses the expression of a gene $g$ through binding to a regulatory element $t$ in cis to the gene, we call such a relationship a regulatory trio. 40 Despite its complexity, gene regulatory logic can be broken down into modular 41 components consisting of such peak-TF-gene trios. In this paper, we focus on the identification of regulatory trios using multiomic experiments that jointly profile gene expression and chromatin accessibility at single-cell resolution.

Single-cell RNA sequencing (scRNA-seq) and single-cell assay of transposaseaccessible chromatin sequencing (scATAC-seq), performed separately, have already generated detailed cell-type-specific profiles of gene expression and chromatin accessibility. When the two modalities are not measured in the same cells, the cells can 48 be aligned by computational methods ${ }^{11}$, followed by association analyses of gene expression and peak accessibility. While these methods have been shown to align well50 differentiated cell types correctly, they often fail for cell populations consisting of transient 51 states. Additionally, the alignment of cells necessarily assumes a peak-gene association 52 model, which is often learned from other datasets. Then, the post-alignment association 
53 analysis is plagued by logical circularity, as it is difficult to disentangle new findings from 54 prior assumptions that underlie the initial cell alignment.

Single-cell multiomic experiments that sequence the RNA and ATAC from the same cells directly enable joint modeling of a cell's RNA expression and chromatin state, yet methods for the analysis of such data are still in their infancy. Almost all existing methods for detecting and characterizing regulatory relationships between TF, regulatory region, and target gene rely on marginal relationships. For example, Signac ${ }^{12}$ and Ma et al. ${ }^{13}$ use marginal associations between peaks and genes to identify putative enhancer regions, while Signac ${ }^{12}$ and Seurat $\mathrm{V} 4{ }^{14}$ link differentially expressed TFs to differentially accessible motifs across cell types. Such pairwise marginal associations are sometimes examined manually, in tandem, using low-dimensional embedding. One exception is in PECA $^{15}$, which uses a parametric model to characterize the joint distribution of TF expression, regulatory site accessibility, chromatin remodeler expression, and target gene expression. Although PECA was designed to be applied to matched bulk transcriptomic and epigenomic data, such joint modeling concepts could potentially be very powerful for single-cell multiomic data. Yet, PECA relies heavily on parametric assumptions, is computationally intensive to fit to large datasets, and is difficult to diagnose.

Context-specific gene regulation may be masked in marginal associations, as we will show in examples later. We explore in this paper the use of higher-order models that interrogate conditional and three-way interaction relationships for the identification of regulatory trios. First, as proof of principle, we show that a simple model that integrates TF expression with cis-peak accessibility significantly improves gene expression prediction, as compared to a comparable model that utilizes peak accessibility alone. We present TRIPOD, a computational framework for transcription regulation interrogation through nonparametric partial association analysis of single-cell multiomic sequencing data. TRIPOD detects two types of trio relationships, which we call conditional level 1 and conditional level 2 , through robust nonparametric tests that are easy to diagnose. A novel

81 influence measure allows the detection and visualization of cell states driving these 82 regulatory relationships, applicable both to discrete cell types, as well as continuous cell 83 trajectories. 
We first apply TRIPOD to single-cell multiomic data of human peripheral blood mononuclear cells (PBMCs) and compare the regulatory trios detected to relationships detected through marginal associations. We show that the detections are coherent with

87 the vast amounts of existing knowledge from enhancer databases, bulk cell-type-specific 88 chromatin immunoprecipitation followed by sequencing (ChIP-seq) experiments, tissuespecific TF knockdown/knockout studies, and cis-eQTL studies, but that conditional and marginal models identify different sets of relationships. We next apply TRIPOD to the

91 interrogation of lineage-specific regulation in the developing mouse brain, where

92 relationships detected by TRIPOD are compared against those derived from existing 93 ChIP-seq and proximity ligation-assisted ChIP-seq (PLAC-seq) data. Here, TRIPOD 94 identifies known trio relationships, as well as putative novel regulatory crosstalk between 95 neuronal TFs and glial-lineage genes. We also apply TRIPOD to SHARE-seq data on 96 mouse hair follicle cell differentiation to illustrate trio detection and influence analysis in 97 data collected from different protocols. Through these analyses, we demonstrate how to 98 harness single-cell multiomic technologies in the study of gene regulation, and how the 99 data from these technologies corroborate and complement existing data.

\section{Results}

102 A simple interaction model between TF expression and peak accessibility improves

103 RNA prediction. We started our analyses by making gene expression predictions, a 104 standard procedure carried out by existing methods ${ }^{11}$. We benchmarked against: (i) 105 Signac $^{12}$ and Cicero $^{16}$, which compute the gene activity matrix by summing the ATAC 106 reads in gene bodies and promoter regions; (ii) MAESTRO ${ }^{17}$, which adopts a regulatory 107 potential model by taking the weighted sum of the ATAC reads based on existing gene 108 annotations; and (iii) sci-CAR ${ }^{18}$, which performs a regularized regression using gene 109 expression as the outcome and peak accessibilities as the predictors. We also use a 110 regularized regression model (referred to as peak-TF LASSO model), where instead of 111 peak accessibility, interactions between TF expressions and peak accessibilities serve as 112 predictors. The model considers only peaks within a certain range of the gene's 113 transcription start site (TSS) and only interactions between TFs and peaks containing 
114 high-scoring binding motifs for the TFs. To avoid overfitting, we perform leave-one-out 115 prediction and adopt independent training and testing sets. See Methods for details.

116 We analyzed single-cell multiomic datasets from different human and mouse

117 tissues generated by different platforms - PBMC by 10X Genomics, embryonic mouse 118 brain by $10 X$ Genomics, mouse skin by SHARE-seq ${ }^{13}$, and adult mouse brain by SNARE$119 \mathrm{seq}^{19}$. Data summaries are included in Supplementary Table 1; reduced dimensions via 120 uniform manifold approximation and projection (UMAP) ${ }^{20}$ are shown in Fig. 1a and 121 Supplementary Fig. 1,2a. To mitigate the undesirable consequences of sparsity and 122 stochasticity in the single-cell data, we clustered cells to form metacells ${ }^{14}$, and pooled 123 gene expression and chromatin accessibility measurements within each metacell.

124 Our results show that, across window sizes, the peak-TF LASSO model 125 significantly improves prediction accuracy across the transcriptome (Fig.1b), with 126 examples shown in (Fig. 1c). This improvement in prediction accuracy holds true when 127 an independent dataset is used for validation (Supplementary Fig. 3). For the SNARE128 seq data ${ }^{19}$, sequencing depth is substantially shallower (Supplementary Fig. 4), thus the 129 improvement of the peak-TF LASSO model is diminished but still evident (Supplementary 130 Fig. 2b). This demonstrates that the product of TF expression and peak accessibility 131 significantly improves RNA prediction accuracy beyond simply using peak accessibility, 132 offering strong empirical evidence of three-way interaction relationships between TF 133 expression, peak accessibility, and target gene expression that can be extracted from 134 such multiomic experiments. However, we will not rely on coefficients from the LASSO 135 model to screen for such trios, as their significance is difficult to compute due to the 136 hazards of post-selection inference ${ }^{21}$. Additionally, accessibility of peaks and expression 137 of TF affecting the same gene are often highly correlated, in which case LASSO tends to 138 select the few with the highest associations and ignore the rest. In such cases, we believe 139 it is more desirable to report all trios.

141 TRIPOD for the detection of peak-TF-gene trio regulatory relationships by single142 cell multiomic data. We propose TRIPOD, a nonparametric method that screens single143 cell RNA and ATAC multiomic data for conditional associations and three-way 144 interactions between the expression of a TF $j$, the accessibility of a peak region 
$145 t$ containing the TF's motif, and the expression of a putative target gene $g$ within a pre146 fixed distance of peak $t$ (Fig. 2a). Existing methods ${ }^{12-14}$ screen for marginal associations 147 either between the TF and the peak or between the peak and the target gene. However, 148 three-way relationships may be complex, and true associations may be masked by 149 population heterogeneity, as we demonstrate later. When marginal associations are 150 masked, evidence for cooperation between the TF and the peak in the regulation of a 151 gene can be inferred from partial associations: (i) with the peak open at a fixed 152 accessibility, whether cells with higher TF expression have higher gene expression; and 153 (ii) with the TF expression fixed at a value above a threshold, whether cells with higher 154 peak accessibility have higher gene expression. To identify such conditional associations 155 without making linearity assumptions on the marginal relationships, TRIPOD matches 156 metacells by either their TF expressions or peak accessibilities (Fig. 2b): For each 157 matched metacell pair, the variable being matched is controlled for, and differences 158 between the pair in the other two variables are computed. Then, across pairs, the 159 nonparametric Spearman's test is used to assess the association between difference in 160 target gene expression $\Delta Y_{g}$ and difference in the unmatched variable (i.e., $\Delta Y_{j}$ if the cells 161 were matched by $X_{t}$, or $\Delta X_{t}$ if the cells were matched by $Y_{j}$ ). We call this the "conditional 162 level 1 test."

163 For illustration, consider the metacell denoted by the black point in Fig. 2b: If we 164 were to match by peak accessibility, this metacell would be matched to the metacell 165 colored in red. We would then compute $\Delta Y_{j}$, the difference between TF $j$ expressions of 166 the matched pair. If we were to match by TF expression, the black dot would be matched 167 to the metacell in green, and we would compute $\Delta X_{t}$, the difference in peak $t$ 168 accessibilities of the pair. In either case, we would compute $\Delta Y_{g}$, the difference in gene $g$ 169 expressions between the pair. We would then mask those metacell-pairs whose values, 170 for the variable being matched, are too low (i.e., those pairs where the TF is off or the 171 peak is closed). Then, $\Delta X_{t}$ or $\Delta Y_{j}$, together with $\Delta Y_{g}$, would be submitted for level 1 test.

172 Even stronger evidence for a regulatory trio could be claimed if the degree of 173 association between the pairwise differences depends on the matched variable. For 174 example, we would tend to believe that TF $j$ binds to peak $t$ to regulate gene $g$ if, in cells 175 with high expression of TF $j$, an increase in peak $t$ accessibility yields a much larger 
176 increase in gene $g$ expression, as compared to in cells with low expression of $\operatorname{TF} j$. One 177 could screen for such interactions by matching by either TF $j$ or peak $t$ accessibility. 178 TRIPOD screens for such interaction effects through a "conditional level 2 test", which 179 assesses the association between $\Delta Y_{g}$ and the product of the matched variable with the 180 difference in the unmatched variable, after taking partial residuals on the difference in the 181 unmatched variable.

182 For significant trios, TRIPOD further carries out a sampling-based influence 183 analysis, where phenotypically contiguous sets of metacells are held out to measure their 184 influence on the estimated coefficients. The corresponding cell types/states that lead to 185 significant deviations from the null upon their removal have high influence scores, which can be used to identify cell types/states that drive a regulatory relationship.

187 To highlight the differences between TRIPOD and existing methods based on 188 marginal associations, we show two canonical examples where the two approaches 189 disagree. Fig. 2c outlines a significant trio detected by TRIPOD's level 2 testing, yet the 190 marginal peak-gene and TF-gene associations were insignificant. It turns out that a 191 subset of cells with high peak accessibility $\left\{X_{t}\right\}$ have close-to-zero TF expressions $\left\{Y_{j}\right\}$, 192 and, meanwhile, another subset of cells with high TF expressions $\left\{Y_{j}\right\}$ have close-to-zero 193 peak accessibilities $\left\{X_{t}\right\}$. In these cells, either the peak is closed, or the TF is not 194 expressed, and this leads to the target gene not being expressed, which masks the 195 marginal associations. The high peak accessibility and TF expression in these cells, 196 which act through other regulatory trios, cancel out when we consider the interaction $197\left\{X_{t} \times Y_{j}\right\}$, leading to a significant interaction term detected by TRIPOD. Conversely, Fig. 198 2d outlines another trio, whose marginal associations were significant, yet TRIPOD did 199 not detect significant conditional associations from either level 1 or level 2 testing. In this 200 case, with almost constant TF expression, the large difference in peak accessibility leads 201 to a small difference in target gene expression. Meanwhile, the cells that drive the 202 significantly positive correlation between $\left\{Y_{g}\right\}$ and $\left\{Y_{j}\right\}$ have almost zero values for $\left\{X_{t}\right\}$. 203 Both observations suggest that this peak has little to do with the regulation of the target 204 gene FGL2 by this specific TF MAFK. Notably, we do not claim that the significantly linked 205 peaks and TFs through marginal association are false positives, but rather this specific 206 trio is insignificant (i.e., the peak and TF may act through other TF and peak, respectively). 
207 In summary, TRIPOD puts peak-TF-gene trios into one unified model, complementing 208 existing methods based on marginal associations and allowing for simultaneous 209 identification of all three factors and prioritization of a different set of regulatory 210 relationships.

212 TRIPOD identifies three-way regulatory relationships in PBMCs with orthogonal

213 validations. We first applied TRIPOD to identify regulatory trios in the 10k PBMC dataset.

214 Cell-type labels for this dataset were transferred from a recently released CITE-seq 215 reference of 162,000 PBMC cells measured with 228 antibodies ${ }^{14}$. After quality control, 216 we kept 7790 cells from 14 cell types pooled into 80 metacells, 103,755 peaks, 14,508 217 genes, and 342 TFs; the UMAP reduced dimensions are shown in Supplementary Fig. 218 1a. Distribution of the number of peaks $100 \mathrm{~kb} / 200 \mathrm{~kb}$ upstream and downstream of the 219 TSS per gene, as well as distribution of the number of motifs per peak, are shown in 220 Supplementary Fig. 5.

221 Results from TRIPOD and marginal association tests overlap but exhibit 222 substantial differences (Supplementary Fig. 6). The previous section showed example 223 trios where the two frameworks disagree. As a proof of concept, we now illustrate two 224 trios where the frameworks agree, identified by level 1 conditional testing (regulation of 225 CCR7 by LEF1, Fig. 3a) and level 2 interaction testing (regulation of GNLY by TBX21, 226 Fig. 3b). From the influence analyses, TRIPOD identified $B$ and T cells as the cell types 227 where LEF1 regulates CCR7, and natural killer (NK) cells as where TBX21 regulates 228 GNLY. These cell type-specific regulatory relationships are corroborated by motif's 229 deviation scores using chromVAR 22 (Fig. 3a,b) and the enrichment of Tn5 integration 230 events in the flanking regions using DNA footprinting analyses ${ }^{12}$ (Supplementary Fig. 7).

231 Unlike chromVar and DNA footprinting analyses, which only give genome-wide average 232 enrichments, TRIPOD significantly enhances the resolution by identifying the specific cis233 regulatory regions that the TFs bind for the regulation of target genes.

234 To our best knowledge, no experimental technique can directly validate three-way 235 regulatory relationships at high resolution with high throughput. Therefore, we performed 236 validation and benchmarking by harnessing existing databases and orthogonal 
237 sequencing experiments that interrogate each pairwise relationship among the three 238 factors (Table 1).

239 First, to validate the cis-linkage between peak region and target gene, we used the 240 enhancer databases of blood and non-cancerous cells from FANTOM5 ${ }^{23}$ (from HACER ${ }^{24}$ ), 2414 DGenome $^{25}$ (from HACER ${ }^{24}$ ), and EnhancerAtlas 2.026, as well as cis-eQTLs in the 242 whole blood reported by the GTEx consortium?. We collapsed TRIPOD's trio calls into 243 peak-gene relationships and benchmarked against Signac's LinkPeaks ${ }^{12}$ on single cells 244 and marginal testing on metacells; for each target gene, we performed a hypergeometric 245 test for enrichment of the peak-gene linkages in the regulatory databases and annotations 246 (see Methods for details). For all four databases, TRIPOD's $p$-values for enrichment are 247 substantially significant (Fig. 3c).

248 Second, to validate the TF-gene edge in the TRIPOD-identified trios, we referred 249 to knockTF ${ }^{7}$, a TF knockdown/knockout gene expression database, and hTFtarget ${ }^{27}$, a 250 database of known TF regulatory targets. Specifically, in knockTF, we found seven TF 251 knockdown/knockout RNA-seq experiments in the peripheral blood category. For these 252 TFs, we identified significantly linked genes by marginal association and by TRIPOD and 253 found TRIPOD's results to have significantly higher precision and recall (Fig. 3d). For 254 hTFtarget, we obtained, for each highly variable gene, its blood-specific TFs, and 255 calculated the gene-specific precision-recall rates - TRIPOD is more sensitive compared 256 to marginal association testing, although both suffered from inflated "false positives," 257 which are likely due to the low sensitivity in the "gold-standard" in silico calls by hTFtarget 258 (Fig. 3e).

259 Third, to validate the TF-peak edge representing TF binding to peak regions, in 260 addition to the DNA footprinting analysis shown in Supplementary Fig. 7e, we 261 downloaded from the Cistrome portal ${ }^{28}$ non-cancerous ChIP-seq data from sorted human 262 blood cells (B lymphocyte, T lymphocyte, and monocyte (Supplementary Table 2). The 263 peaks identified by TRIPOD had a substantially higher percentage of overlap with the 264 ChIP-seq peaks (Fig. 3f). In summary, existing databases and public data of different 265 types from a wide range of studies extensively support each of the three pairwise links in 266 the trios reported by TRIPOD, demonstrating its effectiveness in uncovering true 267 regulatory relationships. 
TRIPOD identifies known and novel putative regulatory relationships during mouse embryonic brain development. We next applied TRIPOD to single-cell multiomic data of $5 \mathrm{k}$ mouse embryonic brain cells at day 18 by $10 \mathrm{X}$ Genomics. The cell type labels were 272 transferred from an independent scRNA-seq reference ${ }^{29}$ using both Seurat V3 ${ }^{11}$ and SAVERCAT ${ }^{30}$. We kept 3,962 cells that had consistent transferred labels from seven major cell types: radial glia, neuroblast, GABAergic neuron, glutamatergic neuron, glioblast, oligodendrocyte, and Cajal-Retzius neuron (Supplementary Fig. 1b). We applied TRIPOD to 633 TFs, 1000 highly variable genes, and ATAC peaks 200kb up/downstream of the genes' TSSs.

First, we investigate a known regulatory trio involving target gene Sox10, TF Olig2 (binding motif CAGCTG), and a cis-regulatory element known as the U2 enhancer (chr15: 79201691-79201880 from $\mathrm{mm} 10$ ) that has been experimentally validated ${ }^{31}$ The expression of Sox10 and Olig2 across the seven cell types, and the ATAC-seq profiles of the region containing the $\mathrm{U} 2$ enhancer and Sox10, are shown in Fig. 4a. This known regulatory trio was found to be conditional level 2 significant by TRIPOD (Fig. 4b). Importantly, the U2 enhancer resides in one of three cis-regulatory elements that were identified by TRIPOD to be enhancers for Sox10 involving TF Olig2; all three significantly linked peaks were validated by Olig2 ChIP-seq data (Fig. 4a).

On the genome scale, the union of TRIPOD's level 1 and 2 tests gave a larger number of unique peak-gene pairs and TF-gene pairs than LinkPeaks ${ }^{12}$ and marginal metacell association tests (Supplementary Fig. 8a). To evaluate results, we first examined whether the peak-gene links were enriched in previously reported enhancer-promoter chromatin contacts using PLAC-seq data of mouse fetal brain ${ }^{32}$ (Table 1, Fig. 4c, Supplementary Fig. 8b). We observed that the regulatory links detected by both marginal association and TRIPOD showed significant enrichment in those supported by PLAC-seq (Fig. 4d, Supplementary Fig. 8c). Importantly, TRIPOD detected sets of trio relationships that were overlapping but distinct from the sets obtained by the marginal model, and a substantial fraction of the links identified by TRIPOD but not by the marginal method were validated by PLAC-seq. This suggests that TRIPOD identifies real regulatory relationships that complement those detected by existing methods. To validate the links between TFs 
299 and peaks, we used publicly available ChIP-seq data of mouse embryonic brain for 300 Olig2 ${ }^{33}$, Neurog $2^{34}$, Eomes ${ }^{34}$, and Tbr135, TFs that play key roles in embryonic brain 301 development (Table 1). TF binding peaks identified by TRIPOD were significantly 302 enriched in the TF ChIP-seq peaks across all embryonic brain datasets; Olig2 ChIP-seq 303 data of mature oligodendrocytes $(\mathrm{mOL})$ serves as a negative control and has insignificant 304 enrichment (Fig. 4e).

305 The validations and global benchmarking demonstrate TRIPOD's effectiveness in 306 finding real regulatory relationships. Next, we focused on a set of TFs known to play 307 essential roles during mouse embryonic brain development. Specifically, we chose Pax6, 308 Neurog2, Eomes, Neurod1, and Tbr1, major TFs mediating glutamatergic neurogenesis ${ }^{36}$, 309 and Olig2, Sox10, Nkx2-2, Sox9, Nfia, and Ascl1, which initiate and mediate gliogenesis ${ }^{37}$; 310 the known regulatory cascade is shown in Fig. 4f. TRIPOD's level 1 and level 2 testing 311 successfully captured six out of the seven known regulatory links; interestingly, TRIPOD's 312 results also suggest substantial crosstalk between the two cascades, where 313 neurogenesis-specific TFs activate gliogenesis-specific TFs (Fig. 4g). ChIP-seq data of

314 Neurog2, Eomes, and Tbr1 supported four of the crosstalk links: regulation of Sox9 by 315 Neurog2 and regulation of Nfia by Neurog2, Eomes, and Tbr1, respectively 316 (Supplementary Fig. 9). These crosstalk links that were validated by ChIP-seq are highly 317 significant by both marginal and conditional associations. Thus, we think it is highly 318 plausible that neurogenesis TFs activate gliogenesis genes at day 18 of embryonic 319 mouse brain development, which is exactly when the switch is being made from 320 neurogenesis to gliogenesis. To our best knowledge, these possible links between 321 neurogenesis and gliogenesis pathways have not been systematically explored and thus 322 warrant future investigation. Finally, for each of the neurogenesis and gliogenesis TFs, 323 we performed a gene ontology (GO) analysis of their significantly linked target genes 324 using DAVID ${ }^{38}$; the enriched terms were largely consistent with the regulatory functions 325 of the TFs during neurogenesis and gliogenesis (e.g., negative regulation of neuron 326 differentiation and oligodendrocyte differentiation) (Fig. 4h).

327 So far, we have taken advantage of the cross-cell-type variation to identify the trio 328 regulatory relationships. To dissect cell-type-specific regulation, we next applied the 329 influence analysis framework (see Methods for details) to the significant trios involving 
330 neurogenesis and gliogenesis TFs. For a given TF, the number of trios, for which a given 331 cell type was influential (FDR < 0.01), is summarized in Fig. 4i, with details for specific 332 example trios given in Supplementary Fig. 10. The analyses underpinned the cell types

333 in which the transcriptional regulation was active, and, reassuringly, the neurogenesis 334 and gliogenesis TFs have the most regulatory influence in neuroblasts and glioblasts, 335 respectively. Additionally, Ascl1 is active in GABAergic neurons in addition to neuroblasts 336 and glioblasts, consistent with its role as a GABAergic fate determinant ${ }^{39}$. Notably, the 337 highly influential cell types that lead to the significant trios involving several neurogenesis338 specific TFs include not only neuroblast but also glioblast, supporting our previous 339 findings on the crosstalk between the two cascades. Overall, TRIPOD allows fine 340 characterization of cell-type- and cell-state-specific functions of the TFs during 341 neurogenesis and gliogenesis.

343 TRIPOD infers lineage-specific regulatory relationships in differentiating mouse 344 hair follicle cells. As a last example, we applied TRIPOD to SHARE-seq ${ }^{13}$ data 345 (Supplementary Fig. 1c) of mouse hair follicle cells, consisting of four broadly defined cell 346 types - transit-amplifying cells (TAC), inner root sheath (IRS), hair shaft, and medulla 347 cells - along a differentiation trajectory. The cell-type labels were curated based on 348 marker genes, TF motifs, and ATAC peaks from the original publication ${ }^{13}$; pseudotime 349 was inferred using Palantir ${ }^{40}$ and overlaid on the cisTopic ${ }^{41}$ reduced dimensions of the 350 ATAC domain. Cells were partitioned using both the pseudotime and the UMAP 351 coordinates to construct metacells (Fig. 5a). Due to the low RNA coverage 352 (Supplementary Fig. 4), we focused on 222 highly-expressed TFs, 794 highly expressed 353 genes reported to have more than ten linked cis-regulatory peaks ${ }^{13}$, and peaks $100 \mathrm{~kb}$ 354 up/downstream of the genes' TSSs.

355 For validation, we used H3K4me1 and H3K27ac ChIP-seq data from an isolated 356 mouse TAC population ${ }^{42}$ (Table 1). H3K4me1 and H3K27ac are marks for active 357 enhancers and are used to benchmark TRIPOD's linked peaks against previously 358 reported domains of regulatory chromatin (DORCs) ${ }^{13}$, as well as randomly sampled peaks.

359 The linked peaks by TRIPOD have higher scores for both H3K4me1 and H3K27ac, than 360 DORCs, the latter identified through marginal associations (Fig. 5b). To further validate 
361 the regulatory effects of the linked peaks, we obtained previously characterized super362 enhancers (SEs) in mouse TACs ${ }^{42}$. Target genes of the 381 SEs were assigned based 363 on the gene's proximity to the SE, as well as the correlation between loss of the SE and 364 loss of the gene transcription ${ }^{42}$. TRIPOD was able to successfully recapitulate the SE 365 regions for the genes considered, with four examples shown in Fig. 5c, where significantly 366 linked peaks mostly reside in the SEs.

367 To demonstrate, Fig. $5 d$ shows regulatory trios that are specific to the IRS lineage, 368 the hair shaft lineage, and the medulla lineage. These trios also show significant pairwise 369 marginal associations (Fig. 5e), lending confidence that they are real. The cell types 370 where the regulation happens are identified by influence analysis, for which the $p$-values 371 are smoothed along the differentiation trajectory and overlaid on the UMAP embedding 372 (Fig. 5f). DNA footprinting analyses surveyed the enrichment of Tn5 integration events 373 surrounding the corresponding motif sites and showed cell-type-specific enrichment (Fig. $3745 \mathrm{~g}$ ), corroborating TRIPOD's results.

\section{Discussion}

377 We have considered the detection of regulatory trios, consisting of a TF binding to a 378 regulatory region to activate or repress the transcription of a nearby gene, using single379 cell RNA and ATAC multiomic sequencing data. The presented method, TRIPOD, is a 380 new nonparametric approach that goes beyond marginal relationships to detect 381 conditional associations and interactions on peak-TF-gene trios. We applied TRIPOD to 382 three single-cell multiomic datasets from different species and protocols with extensive 383 validations and benchmarks. We started our analyses with predicting gene expression 384 from both peak accessibility and TF expression. Supervised frameworks have been 385 proposed to predict gene expression from DNA accessibility ${ }^{43}$, and vice versa ${ }^{44}$, using 386 matched bulk transcriptomic and epigenomic sequencing data. Blatti et al. ${ }^{45}$ showed that 387 joint analysis of DNA accessibility, gene expression, and TF motif binding specificity 388 allows reasonably good prediction of TF binding as measured by ChIP-seq. However, 389 none of these methods incorporate TF expression. By selecting peaks near the genes' 390 TSSs and TFs with high motif scores in the selected peaks, we constructed biologically 
391 meaningful peak-TF pairs as predictors and showed that such a mechanistic model 392 significantly boosts the prediction accuracy of gene expression.

393 We next considered the detection and significance assessment for individual peak-

394 TF-gene trios, comprehensively comparing our detections with those made by tissue- and 395 cell-type-matched PLAC-seq and ChIP-seq experiments, by cis-eQTL and TF 396 knockdown/knockout studies, and by those recorded in the main enhancer databases. 397 Our current study is limited in several ways. A study in Drosophila ${ }^{46}$ modeled motif binding 398 specificities and chromatin accessibilities in bulk RNA and ATAC sequencing data to 399 predict the cooperative binding of pairs of TFs, using in vitro protein-protein binding 400 experiments for validation. The detection of synergies between multiple TFs and peaks 401 on the genome-wide scale and in a cell-type-specific manner needs further investigation. 402 Additionally, while we have not differentiated between positive and negative regulation, 403 TRIPOD reports both types of relationships and categorizes them by sign. While we 404 describe the trios with a positive sign to be enhancers, it is not clear how to interpret the 405 trios with negative signs, the latter having lower overlap with other benchmarking datasets. 406 Transcription activation and repression have been active research areas in biology, with 407 a lot yet unknown ${ }^{47}$. TRIPOD's results provide potential targets for experimental follow408 up and detailed characterization.

409 Our analysis focused on three datasets where the RNA and ATAC modalities have 410 sufficient depths of coverage. For the SHARE-seq data, the sequencing depth for RNA is 411 very low, and thus we focused only on highly expressed genes and TFs (Fig. 5). For 412 SNARE-seq data, whose coverage in both modalities is even lower, we focused on 413 prediction models and not trio detection, where we saw only marginal improvement 414 beyond existing methods ${ }^{19}$ (Supplementary Fig. 2). For data where the coverage is even 415 lower, e.g., PAIRED-seq, cross-modality metacells could not be stably formed, making 416 such analyses impossible (Supplementary Table 1, Supplementary Fig. 4). With rapidly 417 increasing sequencing capacity and technological advancement, TRIPOD, applied to 418 more cells sequenced at higher depth, can uncover novel regulatory relationships at a 419 finer resolution. 


\section{Methods}

422 Data input and construction of metacells. Denote $X_{i t}$ as the peak accessibility for peak

$423 t(1 \leq t \leq T)$ in cell $i(1 \leq i \leq N), Y_{i g}$ as the gene expression for gene $g(1 \leq g \leq G)$, and $424 Y_{i j}$ as the TF expression for TF $j(1 \leq j \leq M)$. The TF expression matrix is a subset of the 425 gene expression matrix, and for single-cell multiomic data, the cell entries are matched. 426 To mitigate the effect of ATAC sparsity ${ }^{48}$ and RNA expression stochasticity ${ }^{49}$, as a first 427 step, TRIPOD performs cell-wise smoothing by pooling similar cells into "metacells." This, 428 by default, is performed using the weighted-nearest neighbor method by Seurat V4 ${ }^{14}$ to 429 jointly reduce dimension and identify cell clusters/states across different modalities. In 430 practice, the metacells can also be inferred using one modality - for example, RNA may 431 better separate the different cell types ${ }^{29}$, and in other cases, chromatin accessibility may 432 prime cells for differentiation ${ }^{13}$. To account for peaks overlapping with other genes 433 (Supplementary Fig. 5b), TRIPOD has the option to either remove the overlapped peaks 434 or to adjust the peak accessibilities by the expressions of the overlapped genes, in a 435 similar fashion to MAESTRO ${ }^{17}$. Library size is adjusted for both the RNA and ATAC 436 domain by dividing all counts by a metacell-specific size factor (total read counts divided 437 by $\left.10^{6}\right)$.

RNA prediction by TF expression and peak accessibility. To predict RNA from ATAC, Signac $^{12}$ and Cicero $^{16}$ take the sum of peak accessibilities in gene bodies and promoter regions to construct a pseudo-gene activity matrix: $\hat{Y}_{i g}=\sum_{t \in E_{g}} X_{i t}$, where $E_{g}$ is the set of

442 peaks within gene bodies and upstream regions of TSSs. Instead of directly taking the 443 sum, MAESTRO ${ }^{17}$ adopts a "regulatory potential" model by taking the weighted sum of 444 accessibilities across all nearby peaks: $\hat{Y}_{i g}=\sum_{t \in E_{g}} w_{t}^{g} X_{i t}$, with weights $\left\{w_{t}^{g}\right\}$ pre445 calculated based on existing gene annotations. Specifically, the method weighs peaks by 446 exponential decay from TSS, sums all peaks on the given gene exons as if they are on 447 the TSS, normalizes the sum by total exon lengths, and excludes the peaks from 448 promoters and exons of nearby genes. The strategy to take the unweighted/weighted sum 449 of accessibility as a proxy for expression has been adopted to align the RNA and ATAC 450 modalities when scRNA-seq and scATAC-seq are sequenced in parallel from the same 451 cell population but not the same cells ${ }^{11}$. For single-cell multiomic data, sci-CAR ${ }^{18}$ performs 
452 feature selection to identify cis-linked peaks via a LASSO regression: $Y_{i g} \sim \sum_{t \in E_{g}} \beta_{t}^{g} X_{i t}$, 453 where an L1 regularization is imposed on $\beta_{t}^{g}$. Compared to MAESTRO, which pre-fixes 454 the weights $\left\{w_{t}^{g}\right\},\left\{\beta_{t}^{g}\right\}$ are estimated from the data by regressing RNA against matched 455 ATAC data. What we propose is a feature selection model involving both peak 456 accessibility and TF expression: $Y_{i g} \sim \sum_{t \in E_{g}} \sum_{j \in f_{t}} \beta_{t j}^{g} X_{i t} Y_{i j}$, where $f_{t}$ contains the set of 457 TFs with high-scoring binding motifs in peak $t$ inferred from the JASPAR database ${ }^{50}$.

TRIPOD model and trio regulatory relationship. For a given target gene $g$, a peak $t$ within a window centered at the gene's TSS, and a TF $j$ whose binding motif is highscoring in the peak, TRIPOD infers the relationship between a regulatory trio $(t, j, g)$. TRIPOD focuses on one trio at a time and goes beyond the marginal associations to characterize the function $Y_{g}=f\left(X_{t}, Y_{j}\right)$. In what follows, we first describe TRIPOD's matching-based nonparametric approach and then describe a linear parametric approach, followed by a discussion on the connections and contrasts between the two approaches.

For each cell $i$ whose TF expression is above a threshold $\delta$ (we only carry out testing in cells that express the TF), we carry out a minimum distance pairwise crossmatch based on $\left\{Y_{i j} \mid Y_{i j}>\delta\right\}$. Let $\left\{\left(i_{p}, i_{p^{*}}\right)\right\}$ be the optimal matching, after throwing away those pairs that have $\left|Y_{i_{p} j}-Y_{i_{p^{*}}}\right|>e$. For each pair $p, i_{p}$ and $i_{p^{*}}$ are two metacells with matched TF expression, for which we now observe two, possibly different, values $\left\{X_{i_{p} t}, X_{i_{p^{*}}}\right\}$ for peak $t$, as well as two corresponding values $\left\{Y_{i_{p} g}, Y_{i_{p^{*}}}\right\}$ for gene $g$. We then compute the following auxiliary differentials within each pair:

$$
\Delta X_{p t}=X_{i_{p} t}-X_{i_{p^{*}}} \text {, }
$$

as well as

$$
\Delta Y_{p g}=Y_{i_{p} g}-Y_{i_{p^{*}}}
$$

477 For level 1 testing of conditional association, we estimate $\hat{r}_{t}^{g}=\rho\left(\Delta X_{p t}, \Delta Y_{p g}\right)$, where $\rho$ is 478 Spearman correlation, and test $H_{1}: r_{t}^{g}=0$. For level 2 testing of interaction, we perform a 479 regression $\Delta Y_{p g}=\alpha \Delta X_{p t}+\gamma \bar{Y}_{p j} \times \Delta X_{p t}$, set $\hat{\gamma}_{t j}$ to be the least-squares solution for $\gamma$, and 480 test $H_{2}: \gamma_{t j}=0$. For visualization of the model fitting, we take the partial residuals of $\Delta Y_{p g}$ 
481 and $\bar{Y}_{p j} \times \Delta X_{p t}$ on $\Delta X_{p t}$, respectively. Note that even though TF expression is not included 482 in the model as a main term, it is controlled for (and not just in the linear sense) by the 483 matching. Similarly, we can also perform this procedure matching by peak accessibility. 484 As a summary, for level 1 testing of conditional association, we have:

Match by $Y_{j}, \alpha=\rho\left(\Delta Y_{g}, \Delta X_{t}\right)$,

Match by $X_{t}, \beta=\rho\left(\Delta Y_{g}, \Delta Y_{j}\right)$.

For level 2 testing of (TF expression) $\times$ (peak accessibility) interaction effects, we have:

$$
\begin{aligned}
& \text { Match by } Y_{j}, \Delta Y_{g}=\alpha^{*} \Delta X_{t}+\gamma_{1}\left(\bar{Y}_{j} \times \Delta X_{t}\right) \text {, } \\
& \text { Match by } X_{t}, \Delta Y_{g}=\beta^{*} \Delta Y_{j}+\gamma_{2}\left(\bar{X}_{t} \times \Delta Y_{j}\right) \text {. }
\end{aligned}
$$

To test for the conditional associations and interactions, we can also use apply a parametric method, such as multiple linear regression:

$$
\begin{gathered}
Y_{g}=\mu+\alpha_{L} X_{t}+\beta_{L} Y_{j}, \\
Y_{g}=\mu+\alpha_{L}^{*} X_{t}+\beta_{L}^{*} Y_{j}+\gamma_{L} X_{t} Y_{j} .
\end{gathered}
$$

See Supplementary Fig. 11 for linear testing results for trios shown in Fig. 3 and Fig. 5. The estimated coefficients from the nonparametric and parametric methods are correlated on the global scale (Supplementary Fig. 12), and their interpretations are similar: $\alpha$ and $\alpha_{L}$ estimate the change in gene expression per change in peak accessibility, fixing TF expression; $\beta$ and $\beta_{L}$ estimate the change in gene expression per change in TF expression, fixing peak accessibility; $\gamma_{1}$ and $\gamma_{L}$ measure how the change in gene expression per change in peak accessibility at each fixed TF expression relies on the TF expression; $\gamma_{2}$ and $\gamma_{L}$ measure how the change in gene expression per change in TF expression at each fixed peak accessibility relies on the peak accessibility. However, the underlying models and assumptions are different. Matching controls for not just the linear variation in the matched variable, but also any nonlinear variation. This contrasts with adding the variable as a covariate in the linear regression, where we simply remove linear dependence. The main motivation for using the matching model above is our reluctance to assume the simple linear relationship. Additionally, we use the rank-based Spearman correlation, which will not be driven by outliers - a "bulk" association between ranks is needed for significance. Thus, the nonparametric model of TRIPOD is more stringent (Supplementary Fig. 13) and more robust to outliers. 
512 Identifying regulatory cell type(s) and cell state(s). For the significant trios detected 513 by TRIPOD, we next seek to identify the underlying regulatory cell type(s). Specifically, 514 we carry out a cell-type-specific influence analysis to identify cell types that are highly 515 influential in driving the significance of the trio. Traditional approaches (e.g., the Cook's 516 distance and the DFFITs) delete observations one at a time, refit the model on remaining 517 observations, and measure the difference in the predicted value from the full model and 518 that from when the point is left out. While they can be readily adopted to detect "influential" 519 metacells one at a time (Supplementary Fig. 7a,b), these methods do not adjust for the 520 degree of freedom properly when deleting different numbers of metacells from different 521 cell types. That is, they do not account for the different numbers of observations that are 522 simultaneously deleted. Additionally, both methods adopt a thresholding approach to 523 determine significance, without returning $p$-values that are necessary for multiple testing 524 correction. We, therefore, develop a sampling-based approach to directly test for the influence of multiple metacells and to return $p$-values (Supplementary Fig. 7c).

Here, we focus on the linear model for its ease of computation: $\hat{Y}_{g}=\hat{\mu}+\hat{\alpha} X_{t}+$ $\hat{\beta} Y_{j}+\hat{\gamma} X_{t} Y_{j}$. Given a set of observations $I=\{i: i$ th metacell belongs to a cell type $\}$, we remove these metacells, fit the regression model, and make predictions: $\hat{Y}_{g}^{(I)}=\hat{\mu}^{(I)}+$ $\hat{\alpha}^{(I)} X_{t}+\hat{\beta}^{(I)} Y_{j}+\hat{\gamma}^{(I)} X_{t} Y_{j}$. The test statistics are the difference in the fitted gene expressions $\left|\widehat{Y}_{g}-\widehat{Y}_{g}^{(I)}\right|$. We generate the null distribution via sampling. Specifically, within each sampling iteration, we sample without replacement the same number of metacells, 532 denoted as a set of $I^{*}$, delete these observations, and refit the regression model on the 533 remaining observations: $\hat{Y}_{g}^{\left(I^{*}\right)}=\hat{\mu}^{\left(I^{*}\right)}+\hat{\alpha}^{\left(I^{*}\right)} X_{t}+\hat{\beta}^{\left(I^{*}\right)} Y_{j}+\hat{\gamma}^{\left(I^{*}\right)} X_{t} Y_{j}$. The $p$-value is 534 computed across $K$ sampling iterations as $p_{Y_{g}}=\sum_{I^{*}} 1\left(\sum\left|\hat{Y}_{g}-\hat{Y}_{g}^{(I)}\right| \geq \sum\left|\hat{Y}_{g}-\hat{Y}_{g}^{\left(I^{*}\right)}\right|\right) / K$, 535 where 1() is the indicator function. In addition to testing each cell type separately, the 536 framework can be extended to test for the influence of cell-type groups. For example, in 537 Fig. 3a,b, we reconstruct the cell-type hierarchy using expression levels of highly variable 538 genes from the RNA domain and carry out the aforementioned testing scheme at each 539 split for its descendent cell types in the hierarchical structure. 
For transient cell states, TRIPOD first identifies the neighbors of each metacell along the trajectory and then carries out metacell-specific testing by simultaneously removing each metacell and its neighbors using the framework described above. The resulting $p$-values are, therefore, smoothed and can be visualized in the UMAP plot, as shown in Fig. $5 f$ and Supplementary Fig. 10, to identify the underlying branches/segments that are key in defining the significant regulatory trio. This approach can be directly applied to cells with branching dynamics without the need to isolate cell subsets or to identify cell types.

Validation resources and strategies. Resources for validating the trio regulatory relationships are summarized in Table 1. To validate the peak-gene relationships, we referred to existing enhancer databases: FANTOM5 ${ }^{23}$ links enhancers and genes based on enhancer RNA expression; 4DGenome ${ }^{25}$ links enhancers and genes based on physical interactions using chromatin-looping data including 3C, 4C, 5C, ChIA-PET, and $\mathrm{Hi}-\mathrm{C}$; EnhancerAtlas $2.0^{26}$ reports enhancers using 12 high-throughput experimental methods including $\mathrm{H} 3 \mathrm{~K} 4 \mathrm{me} 1 / \mathrm{H} 3 \mathrm{~K} 27$ ac ChIP-seq, DNase-seq, ATAC-seq, and GRO-seq. We only focused on blood and non-cancerous cells from these databases (Fig. 3c). A list of cis-eQTLs within the whole blood mapped in European-American subjects was downloaded from the GTEx consortium ${ }^{9}$ (Fig. 3c). For the mouse embryonic brain dataset, we additionally adopted H3K4me3-mediated PLAC-seq data ${ }^{32}$, which reported enhancerpromoter chromatin contacts mapped in mouse fetal forebrain (Fig. 4c,d). For the mouse skin dataset, we adopted TAC-specific ChIP-seq data of H3K4me1 and $\mathrm{H} 3 \mathrm{~K} 27 \mathrm{ac}^{42}$, both of which are histone marks for active enhancers (Fig. 5b); we also obtained previously reported super-enhancers in mouse TACs from in vivo studies ${ }^{42}$ (Fig. 5c). Genomic coordinates were lifted over from $\mathrm{mm} 9$ to $\mathrm{mm} 10$ when necessary.

To validate the TF-gene relationships, we utilized the knockTF ${ }^{7}$ and the hTFtarget ${ }^{27}$ databases. knockTF interrogates the changes in gene expression profiles in TF 567 knockdown/knockout experiments to link the TFs to their target genes in a tissue- or cell568 type-specific manner. We downloaded 12 experiments, corresponding to 12 TFs 569 (BCL11A, ELK1, GATA3, JUN, MAF, MYB, NFATC3, NFKB1, STAT3, STAT6, TAL1, and 570 
571 one linked gene by any model benchmarked (Fig. 3d). hTFtarget computationally predicts 572 TF-gene relationships using ChIP-seq data, and we manually downloaded the TFs 573 associated with each of the top 100 highly variable genes in the blood tissue (Fig. 3e).

574 To validate the peak-TF relationships, we downloaded non-cancerous cell-type575 specific ChIP-seq data of human blood (B lymphocyte, T lymphocyte, and monocyte) from 576 the Cistrome ${ }^{28}$ portal for the PBMC data (Fig. 3f, Supplementary Table 2), and ChIP-seq 577 data of Olig2 ${ }^{33}$, Neurog $2^{34}$, Eomes ${ }^{34}$, and Tbr $1^{35}$ for the mouse embryonic brain data. The 578 Olig2 ChIP-seq data were generated in three types of rat cells: data from oligodendrocyte precursor cells (OPC) and immature oligodendrocytes (iOL) were used for validation,

580 while data from mature oligodendrocytes $(\mathrm{mOL})$ serve as a negative control ${ }^{33}$. Genomic 581 coordinates were converted from rn4 to mm10. The Neurog2 and Eomes ChIP-seq data were generated in mouse embryonic cerebral cortices at day 14.534; the Tbr1 ChIP-seq 583 data was generated in the whole cortex dissected from embryos at day $15.5^{35}$. In addition, 584 DNA footprinting signatures were corrected for Tn5 sequence insertion bias and stratified by cell types using the Signac package and can be used to validate the identified TFs/motifs in a cell-type-specific manner (Fig. 5g, Supplementary Fig. 7e).

For peak enrichment analysis compared to the existing enhancers, cis-eQTLs, and enhancer-promoter contacts, we carried out a hypergeometric test as follows. Let $k$ be the number of significantly linked peaks, $q$ be the number of significantly linked peaks that overlap with annotations (e.g., annotated enhancers), $m$ be the number of peaks that overlap with the annotations, and $n$ be the number of peaks that do not overlap with 592 annotations. The $p$-value of enrichment is derived from the hypergeometric distribution 593 using the cumulative distribution function, coded as phyper(q, m, n, k, lower.tail=F) in R.

594 We used this hypothesis testing framework to validate and benchmark the reported peak595 gene links, with results shown in Fig. 3c and Fig. 4d. A similar analysis was carried out to 596 test for peak enrichment in TF binding sites by ChIP-seq, thus validating the peak-TF 597 relationships (Fig. 4e).

599 Data availability

600 This study analyzed existing and publicly available single-cell RNA and ATAC multiomic 601 data. 10X Genomics single-cell multiomic datasets of PBMC (10k and 3k) and mouse 
602 embryonic brain were downloaded https://support.10xgenomics.com/single-cell-

603 multiome-atac-gex/datasets. SNARE-seq data of adult mouse brain and SHARE-seq 604 data of mouse skin are available from the Gene Expression Omnibus (GEO) database 605 with accession numbers GSE126074 and GSE140203. A detailed data summary is 606 provided in Supplementary Table 1. Validation resources based on existing databases 607 and high-throughput sequencing data are summarized in Table 1 and Supplementary 608 Table 2.

610 Code availability

611 TRIPOD is compiled as an open-source $R$ package available at 612 https://github.com/yharigaya/TRIPOD. Scripts used for analyses carried out in this paper 613 are deposited in the GitHub repository.

614

\section{Acknowledgments}

616 This work was supported by the National Institutes of Health (NIH) grant R35 GM133712 617 (to C.Z.), R01 HG006137 (to N.R.Z), R01 GM125301 (to N.R.Z.), and R35 GM138342 (to 618 Y.J.). The authors thank Dr. Sai Ma for support and guidance on the SHARE-seq data, 619 Manas Tiwari for help on accessing the hTFtarget database, and Drs. Yun Li, Michael 620 Love, Li Qian, and Jason Stein for helpful discussions and comments.

\section{Author contributions}

623 N.R.Z. and Y.J. initiated and envisioned the study. Y.H., N.R.Z., and Y.J. formulated the 624 model, developed the algorithm, and performed data analysis. Z.Z. processed reference 625 datasets and performed cell-type label transfer. H.Z. and C.Z. provided support on 626 validation, offered consultation, and contributed to result interpretation. Y.H., N.R.Z., and 627 Y.J. wrote the manuscript, which was read and approved by all authors.

\section{Competing Interests}

630 The authors declare no competing interests. 


\section{Figure Legends}

633 Fig. 1 | Interaction between TF expression and peak accessibility improves RNA 634 prediction accuracy. a, UMAP embedding of 10x Genomics PBMC (left), 10x Genomics

635 embryonic mouse brain (center), and SHARE-seq mouse skin (right) cells from single636 cell RNA and ATAC multiomic sequencing. Cell-type labels were transferred from existing 637 single-cell references or curated based on marker genes, motifs, and peaks; metacells 638 were constructed to mitigate sparsity and stochasticity. b. Genome-wide distributions of 639 Pearson correlations between observed and leave-one-out predicted RNA expression 640 levels, with varying window sizes. c, Predicted and observed RNA expression levels for 641 highly variable genes, CCR7, Adamts6, and Ano7, from the three datasets, respectively.

Fig. 2 | TRIPOD infers peak-TF-gene trio regulatory relationships using single-cell multiomic data. a, Data input and schematic on a peak-TF-gene trio. $\mathbf{b}$, Overview of TRIPOD for inferring regulatory relationships. TRIPOD complements existing methods 646 based on marginal associations by identifying conditional associations through matching 647 by TF expression or peak accessibility. c, An example trio identified by TRIPOD, but not 648 by the marginal associations due to heterogeneity of cell-type-specific regulations. $\mathbf{d}$, An 649 example trio identified by the marginal associations, but not by TRIPOD. The peak and 650 TF are significantly linked to the gene, yet they act through other TF and peak, and thus 651 the regulatory trio is insignificant. The points represent metacells (left two panels) and 652 pairs of matched metacells (right two panels). Genomic coordinates for the peaks are 653 from hg38.

655 Fig. 3 | TRIPOD identified trio regulatory relationships in PBMC single-cell 656 multiomic dataset. a-b, Example trios identified by TRIPOD. Violin plots show cell-type657 specific distributions of gene expression, peak accessibility, and TF expression. 658 Scatterplots show TRIPOD's level 1 and level 2 testing, respectively. Inner and outer 659 circles around the points are color-coded based on the cell types of the matched metacells. 660 Hierarchical clustering is performed on RNA expression levels of highly variable genes. 661 Red/gray circles indicate whether removal of the corresponding branches of metacells 662 significantly changes the model fitting; crosses indicate that removal of the groups of 
663 metacells resulted in inestimable coefficients. Genomic coordinates for the peaks are 664 from hg38. c, Peak-gene validation based on enhancer databases (FANTOM5, 665 4DGenome, and EnhancerAtlas) and tissue-specific cis-eQTL data from the GTEx 666 Consortium. Box plots show distributions of $p$-values from gene-specific hypergeometric 667 tests. d, TF-gene validation based on lists of TF-gene pairs from the knockTF database. $668 \mathrm{e}$, Precision and recall rates for TF-gene pairs using ground truths from the hTFtarget 669 database. f, Peak-TF validation based on eight cell-type-specific TF ChIP-seq datasets 670 (B lymphocytes, monocytes, and $\mathrm{T}$ lymphocytes). Percentages of significantly linked 671 peaks and all peaks that overlap with the ChIP-seq peaks are shown. Fig. 4 | TRIPOD identified known and novel regulatory relationships during mouse neurogenesis and gliogenesis. a, TRIPOD identified a previously reported regulatory trio with gene Sox10, TF Olig2, and cis-regulatory U2 element. TRIPOD identified two additional linked peaks; all three cis-regulatory elements were validated by Olig2 ChIPseq data. b, TRIPOD's level 2 testing matching peak accessibility for the Sox10 gene, the Olig2 TF, and the U2 enhancer. c, Venn diagram of the number of peak-gene pairs captured by PLAC-seq, the marginal model, and the union set of TRIPOD's level 1 and level 2 testing matching TF expression. d, Enrichment of peak-gene pairs captured by LinkPeaks, marginal association, and TRIPOD in enhancer-promoter contacts by PLACseq. e, Peak-TF validation by ChIP-seq data. Olig2 ChIP-seq data of precursor/immature oligodendrocytes (OPC/iOL) were used for validation; data from mature oligodendrocytes ( $\mathrm{mOL}$ ) served as a negative control. $\mathbf{f}$, A schematic of well-characterized TF regulatory cascades during neurogenesis and gliogenesis. $\mathbf{g}$, Trio examples from known regulatory relationships, as well as from crosstalks supported by ChIP-seq data, captured by TRIPOD. $\mathbf{h}, \mathrm{GO}$ analysis of putative target genes of the neurogenesis and gliogenesis TFs. The number of TRIPOD-identified target genes in the GO categories is shown. The background heatmap shows negative log $p$-values (FDR adjusted) from hypergeometric tests examining enrichment of ATAC peaks in ChIP-seq peaks. i, Bar plots showing the 691 number of putative cell-type-specific trios mediated by the neurogenesis- and gliogenesis692 specific TFs.

693 
694 Fig. 5 | TRIPOD identified regulatory relationships in mouse hair follicles with

695 transient cell states. a, UMAP embedding of hair follicle cells from the mouse skin data.

696 Cells are colored by cell types (TAC, IRS, hair shaft, and medulla) and pseudotime. b,

697 H3K4me1 and H3K27ac ChIP-seq scores for linked peaks identified by TRIPOD, DORCs

698 (regulatory domains identified by gene-peak correlations), and randomly sampled peaks.

699 c, TRIPOD's linked peaks for four representative genes were significantly enriched in

700 previously annotated super-enhancers in the mouse TAC population. $\mathbf{d}$, Trios detected

701 by TRIPOD that were active in IRS (top), medulla (middle), and hair shaft (bottom),

702 respectively. e, Dot plots of gene expressions, peak accessibilities, and TF expressions

703 across different cell types. f, Influence analyses identified segments along the

704 differentiation trajectory where the regulation took effect. The colors in the UMAP

705 embedding correspond to the smoothed $p$-values from a sampling-based approach. $\mathbf{g}$,

706 DNA footprinting assays showed cell-type-specific enrichments of Tn5 integration events.

707 The findings were consistent with those from the influence analyses.

Table 1 | Resources for validating peak-TF-gene regulatory relationship. While there

710 is no existing experimental approach to validate all three factors in a trio at high resolution

711 with high throughput, we resort to existing databases and orthogonal sequencing data to

712 validate peak-gene, peak-TF, and TF-gene pairs, completing the loop.

\section{References}

715 1. Gasperini, M., Tome, J.M. \& Shendure, J. Towards a comprehensive catalogue of validated and target-linked human enhancers. Nat Rev Genet 21, 292-310 (2020).

2. Hobert, O. Gene regulation by transcription factors and microRNAs. Science 319, 1785-1786 (2008).

3. Mortazavi, A., Williams, B.A., McCue, K., Schaeffer, L. \& Wold, B. Mapping and quantifying mammalian transcriptomes by RNA-Seq. Nat Methods 5, 621-628 (2008).

4. Johnson, D.S., Mortazavi, A., Myers, R.M. \& Wold, B. Genome-wide mapping of in vivo proteinDNA interactions. Science 316, 1497-1502 (2007).

5. Buenrostro, J.D., Giresi, P.G., Zaba, L.C., Chang, H.Y. \& Greenleaf, W.J. Transposition of native chromatin for fast and sensitive epigenomic profiling of open chromatin, DNA-binding proteins and nucleosome position. Nat Methods 10, 1213-1218 (2013).

6. Lieberman-Aiden, E. et al. Comprehensive mapping of long-range interactions reveals folding principles of the human genome. Science 326, 289-293 (2009).

7. Feng, C. et al. KnockTF: a comprehensive human gene expression profile database with knockdown/knockout of transcription factors. Nucleic Acids Res 48, D93-D100 (2020). 
8. Tompa, M. et al. Assessing computational tools for the discovery of transcription factor binding sites. Nat Biotechnol 23, 137-144 (2005).

733

9. Consortium, G. Genetic effects on gene expression across human tissues. Nature 550, 204-213 (2017).

10. Kim-Hellmuth, S. et al. Cell type-specific genetic regulation of gene expression across human tissues. Science 369 (2020).

11. Stuart, T. et al. Comprehensive Integration of Single-Cell Data. Cell 177, 1888-1902 e1821 (2019).

12. Stuart, T., Srivastava, A., Lareau, C. \& Satija, R. Multimodal single-cell chromatin analysis with Signac. bioRxiv, 2020.2011.2009.373613 (2020).

13. Ma, S. et al. Chromatin Potential Identified by Shared Single-Cell Profiling of RNA and Chromatin. Cell 183, 1103-1116 e1120 (2020).

741 14. Hao, Y. et al. Integrated analysis of multimodal single-cell data. Cell 184, 3573-3587 e3529 (2021).

15. Duren, Z., Chen, X., Jiang, R., Wang, Y. \& Wong, W.H. Modeling gene regulation from paired expression and chromatin accessibility data. Proc Natl Acad Sci U S A 114, E4914-E4923 (2017).

16. Pliner, H.A. et al. Cicero Predicts cis-Regulatory DNA Interactions from Single-Cell Chromatin Accessibility Data. Mol Cell 71, 858-871 e858 (2018).

17. Wang, C. et al. Integrative analyses of single-cell transcriptome and regulome using MAESTRO. Genome Biol 21, 198 (2020).

18. Cao, J. et al. Joint profiling of chromatin accessibility and gene expression in thousands of single cells. Science 361, 1380-1385 (2018).

19. Chen, S., Lake, B.B. \& Zhang, K. High-throughput sequencing of the transcriptome and chromatin accessibility in the same cell. Nat Biotechnol 37, 1452-1457 (2019).

20. Becht, E. et al. Dimensionality reduction for visualizing single-cell data using UMAP. Nat Biotechnol (2018).

21. Taylor, J. \& Tibshirani, R.J. Statistical learning and selective inference. Proc Natl Acad Sci U S A 112, 7629-7634 (2015).

22. Schep, A.N., Wu, B., Buenrostro, J.D. \& Greenleaf, W.J. chromVAR: inferring transcription-factorassociated accessibility from single-cell epigenomic data. Nat Methods 14, 975-978 (2017).

23. Andersson, R. et al. An atlas of active enhancers across human cell types and tissues. Nature 507, 455-461 (2014).

24. Wang, J. et al. HACER: an atlas of human active enhancers to interpret regulatory variants. Nucleic Acids Res 47, D106-D112 (2019).

25. Teng, L., He, B., Wang, J. \& Tan, K. 4DGenome: a comprehensive database of chromatin interactions. Bioinformatics 32, 2727 (2016).

26. Gao, T. \& Qian, J. EnhancerAtlas 2.0: an updated resource with enhancer annotation in 586 tissue/cell types across nine species. Nucleic Acids Res 48, D58-D64 (2020).

27. Zhang, Q. et al. hTFtarget: A Comprehensive Database for Regulations of Human Transcription Factors and Their Targets. Genomics Proteomics Bioinformatics 18, 120-128 (2020).

28. Mei, S. et al. Cistrome Data Browser: a data portal for ChIP-Seq and chromatin accessibility data in human and mouse. Nucleic Acids Res 45, D658-D662 (2017).

29. La Manno, G. et al. Molecular architecture of the developing mouse brain. Nature 596, 92-96 (2021).

30. Huang, M., Zhang, Z. \& Zhang, N.R. Dimension reduction and denoising of single-cell RNA sequencing data in the presence of observed confounding variables. bioRxiv, 2020.2008.2003.234765 (2020).

31. Kuspert, M., Hammer, A., Bosl, M.R. \& Wegner, M. Olig2 regulates Sox10 expression in oligodendrocyte precursors through an evolutionary conserved distal enhancer. Nucleic Acids Res 39, 1280-1293 (2011). 
32. Zhu, C. et al. An ultra high-throughput method for single-cell joint analysis of open chromatin and transcriptome. Nat Struct Mol Biol 26, 1063-1070 (2019).

33. $\mathrm{Yu}, \mathrm{Y}$. et al. Olig2 targets chromatin remodelers to enhancers to initiate oligodendrocyte differentiation. Cell 152, 248-261 (2013).

34. Sessa, A. et al. The Tbr2 Molecular Network Controls Cortical Neuronal Differentiation Through Complementary Genetic and Epigenetic Pathways. Cereb Cortex 27, 5715 (2017).

35. Notwell, J.H. et al. TBR1 regulates autism risk genes in the developing neocortex. Genome Res 26, 1013-1022 (2016).

36. Mira, H. \& Morante, J. Neurogenesis From Embryo to Adult - Lessons From Flies and Mice. Front Cell Dev Biol 8, 533 (2020).

37. Emery, B. \& Lu, Q.R. Transcriptional and Epigenetic Regulation of Oligodendrocyte Development and Myelination in the Central Nervous System. Cold Spring Harb Perspect Biol 7, a020461 (2015).

38. Huang da, W., Sherman, B.T. \& Lempicki, R.A. Systematic and integrative analysis of large gene lists using DAVID bioinformatics resources. Nat Protoc 4, 44-57 (2009).

39. Achim, K., Salminen, M. \& Partanen, J. Mechanisms regulating GABAergic neuron development. Cell Mol Life Sci 71, 1395-1415 (2014).

40. Setty, M. et al. Characterization of cell fate probabilities in single-cell data with Palantir. Nat Biotechnol 37, 451-460 (2019).

41. Bravo Gonzalez-Blas, C. et al. cisTopic: cis-regulatory topic modeling on single-cell ATAC-seq data. Nat Methods 16, 397-400 (2019).

42. Adam, R.C. et al. Pioneer factors govern super-enhancer dynamics in stem cell plasticity and lineage choice. Nature 521, 366-370 (2015).

43. Natarajan, A., Yardimci, G.G., Sheffield, N.C., Crawford, G.E. \& Ohler, U. Predicting cell-typespecific gene expression from regions of open chromatin. Genome Res 22, 1711-1722 (2012).

44. Zhou, W. et al. Genome-wide prediction of DNase I hypersensitivity using gene expression. Nat Commun 8, 1038 (2017).

45. Blatti, C., Kazemian, M., Wolfe, S., Brodsky, M. \& Sinha, S. Integrating motif, DNA accessibility and gene expression data to build regulatory maps in an organism. Nucleic Acids Res 43, 3998-4012 (2015).

46. Kazemian, M., Pham, H., Wolfe, S.A., Brodsky, M.H. \& Sinha, S. Widespread evidence of cooperative DNA binding by transcription factors in Drosophila development. Nucleic Acids Res 41, 8237-8252 (2013).

47. Panigrahi, A. \& O'Malley, B.W. Mechanisms of enhancer action: the known and the unknown. Genome Biol 22, 108 (2021).

48. Urrutia, E., Chen, L., Zhou, H. \& Jiang, Y. Destin: toolkit for single-cell analysis of chromatin accessibility. Bioinformatics 35, 3818-3820 (2019).

49. Jiang, Y., Zhang, N.R. \& Li, M. SCALE: modeling allele-specific gene expression by single-cell RNA sequencing. Genome Biol 18, 74 (2017).

50. Fornes, O. et al. JASPAR 2020: update of the open-access database of transcription factor binding profiles. Nucleic Acids Res 48, D87-D92 (2020). 
Figures
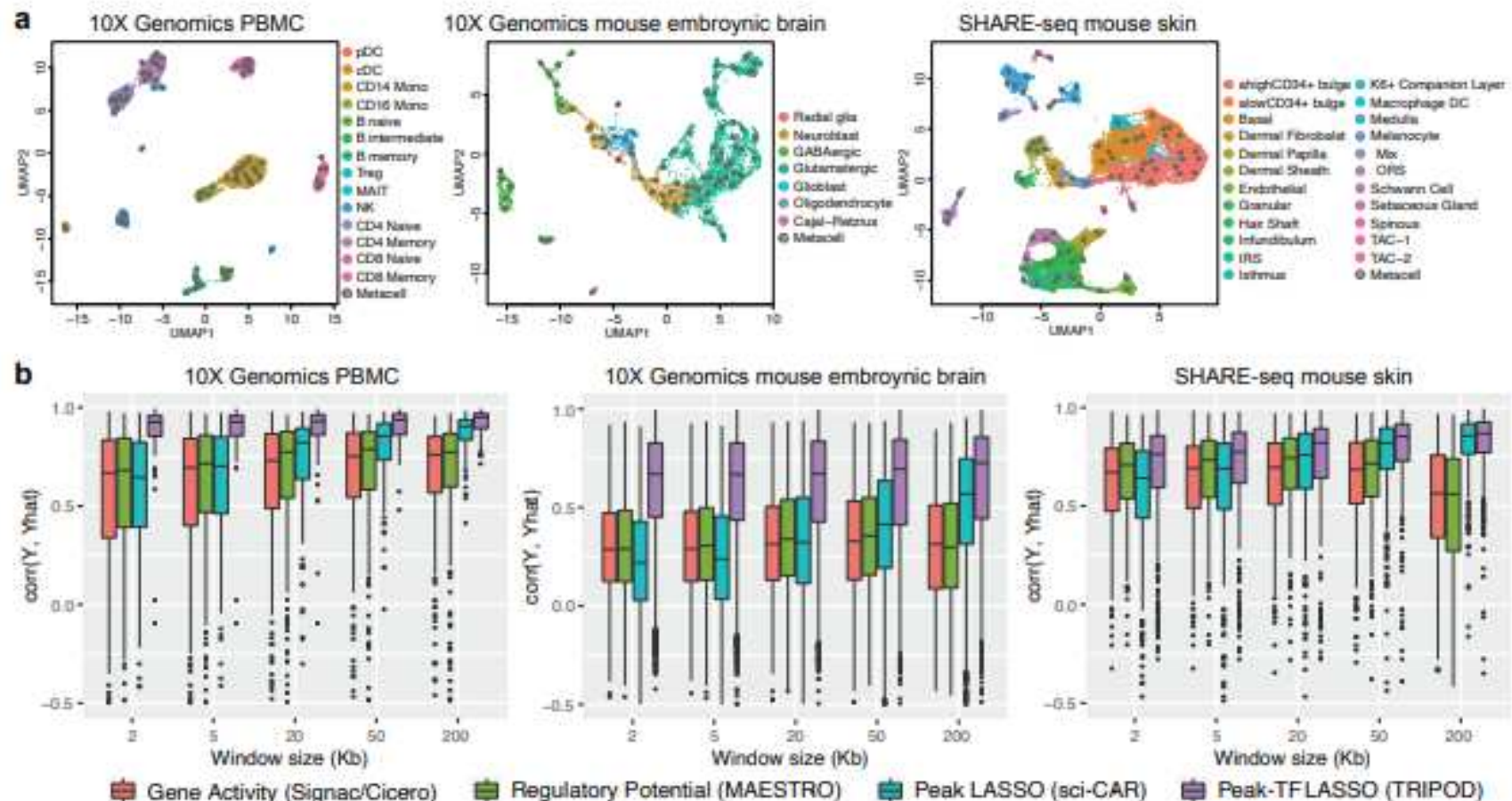

c

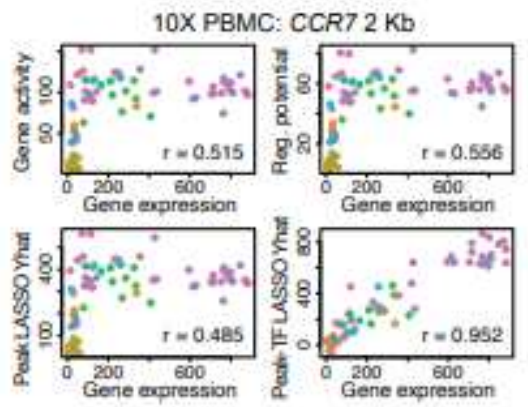

Regulatory Potential (MAESTRO) 鼻 Peak LASSO (sci-CAR)

Peak-TFLASSO (TRIPOD)

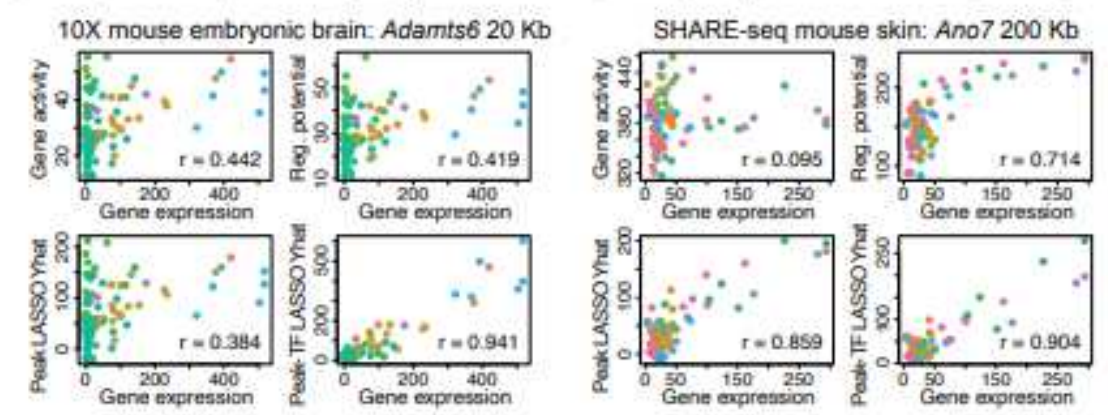

Figure 1

\section{Figure 1}

Interaction between TF expression and peak accessibility improves RNA prediction accuracy. 
a

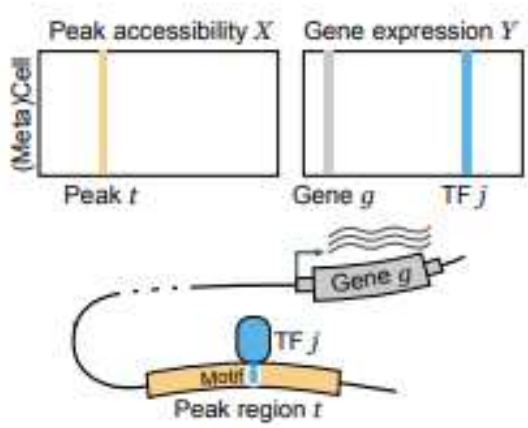

b

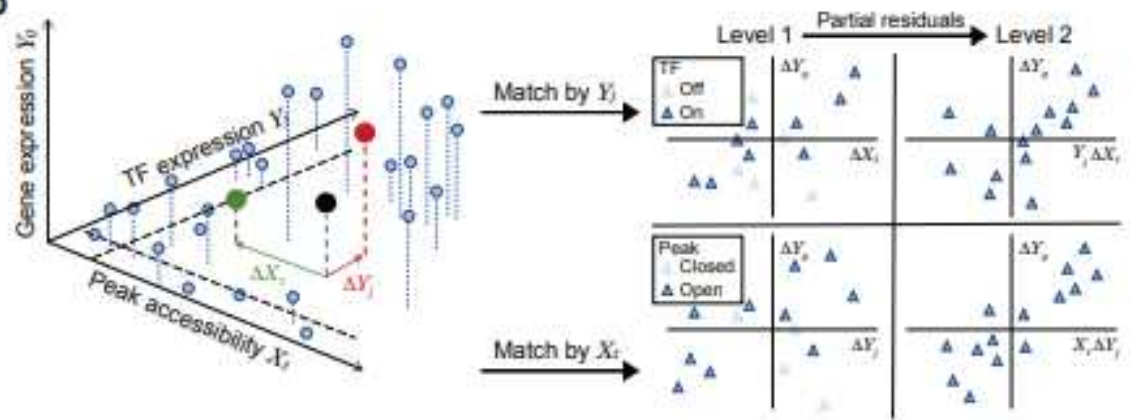

C

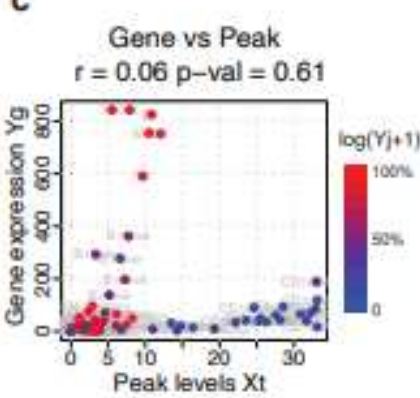

d

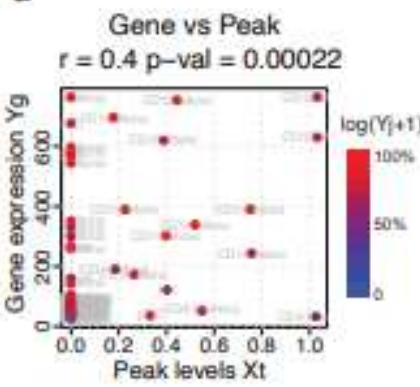

Gene ZBTB16, Peak chr11-114134804-114137698, TF RORA

Gene vs TF TRIPOD match by $Y$ J conditional

$r=0.28 p-v a l=0.013$

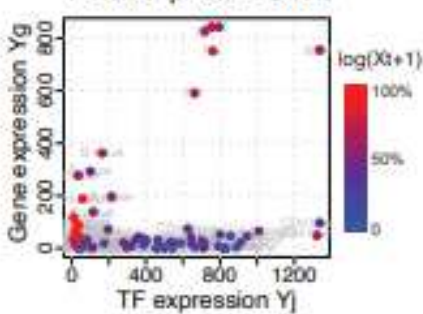

TRIPOD match by $Y$ j interaction

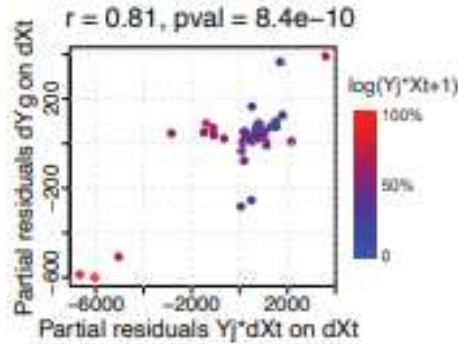

Gene FGL.2, Peak chr7-77195757-77195901, TF MAFK

Gene vs TF

$$
f=0.39 p-v a l=0.00039
$$

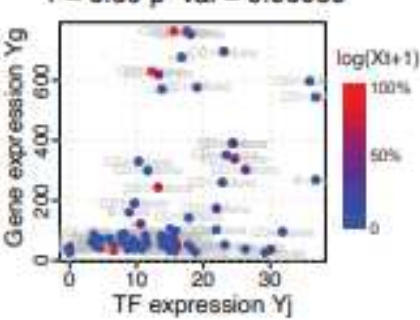

TRIPOD match by $Y j$ conditional

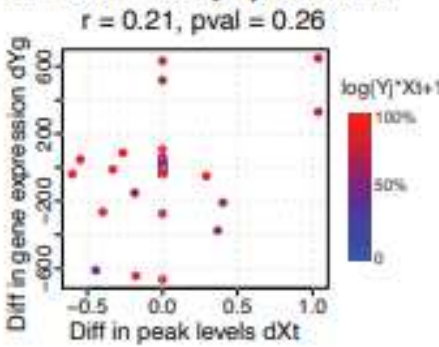

TRIPOD match by $Y$ j interaction

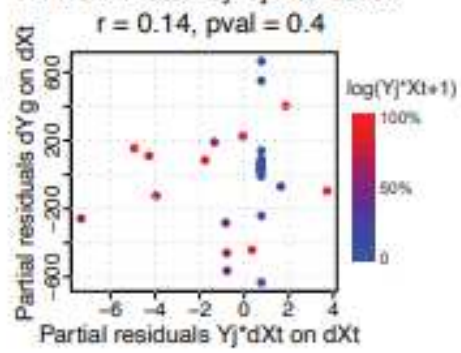

Figure 2

TRIPOD infers peak-TF-gene trio regulatory relationships using single-cell multiomic data. 

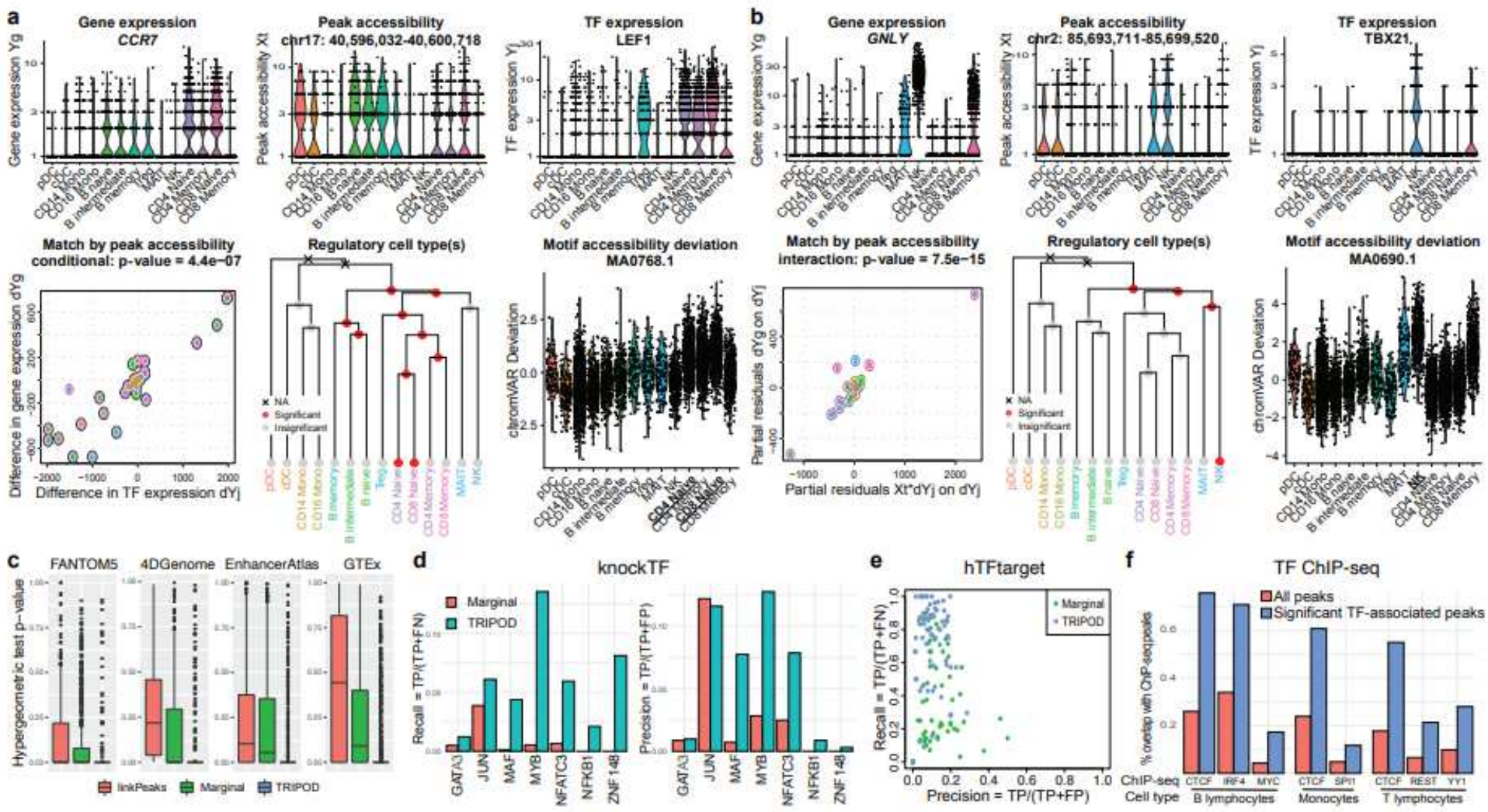

Figure 3

Figure 3

TRIPOD identified trio regulatory relationships in PBMC single-cell multiomic dataset. 
a
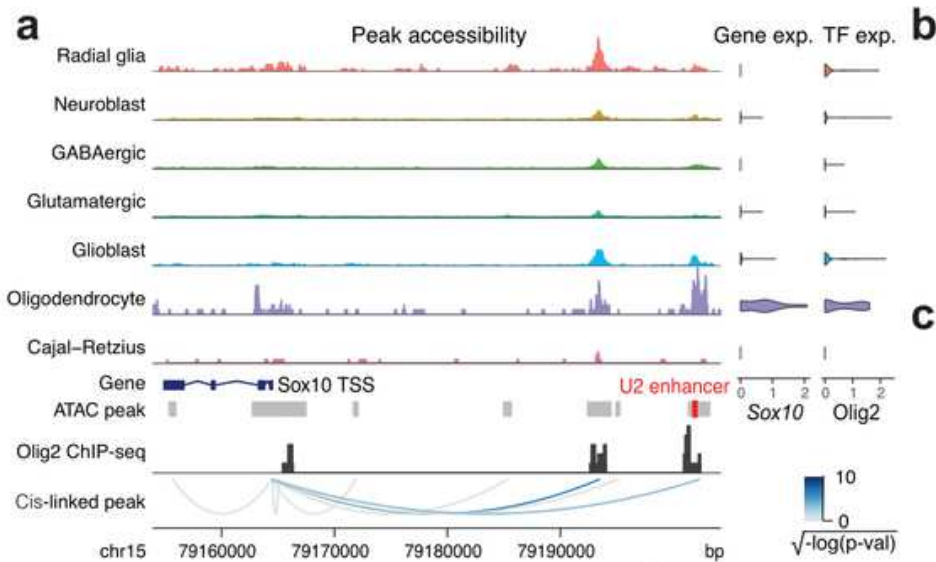

f

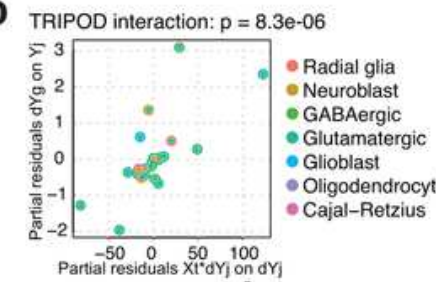

C Marginal TRIPOD $\mathbf{d}_{\text {Peak-gene validation by PLAC-seq }}$

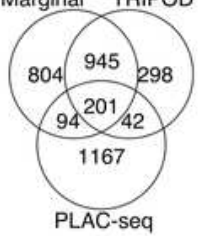

$\begin{array}{lll}2.94 & 2.32 & 3.07 \\ 2 & \text { LinkPeaks }\end{array}$

14.4615 .1317 .36 Marginal

17.1419 .4221 .06 TRIPOD

9.80 13.31 14.47 Marginal + TRIPOD

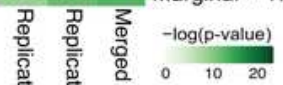

e

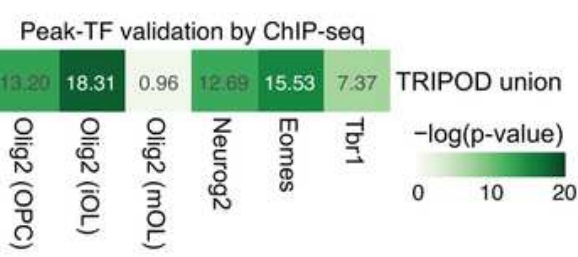

Pax6 Neurog2 Eomes Neurod1 Tbr1 Neurogenesis

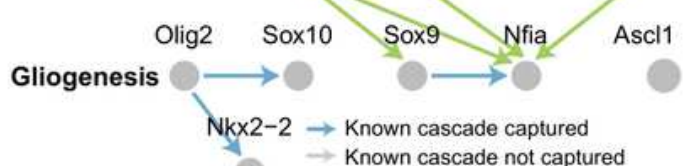

$\rightarrow$ Known cascade not captured

$\rightarrow$ Crosstalk captured and supported by ChIP-seq

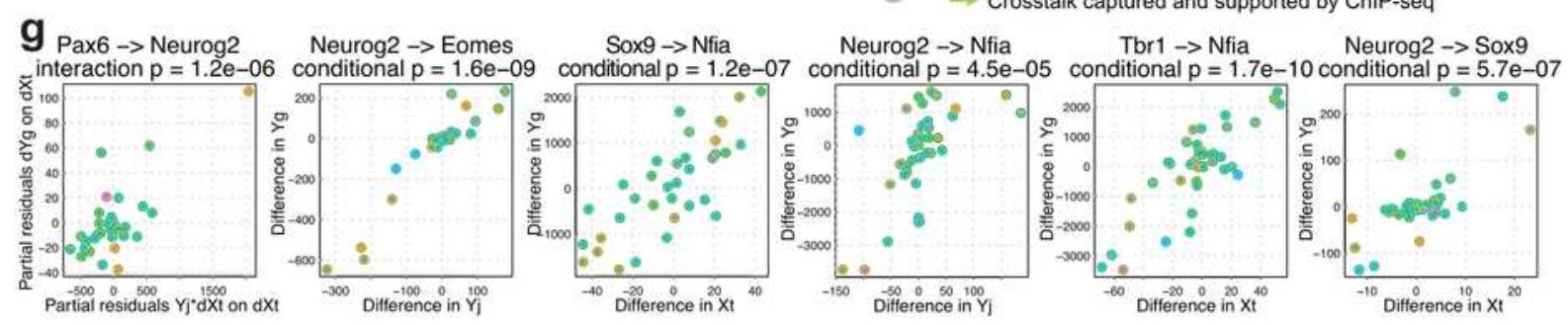

h

\begin{tabular}{|c|c|c|c|c|c|c|c|c|c|c|c|}
\hline 20 & 14 & 12 & 16 & 8 & 17 & NA & NA & 25 & 16 & 15 & regulation of transcription from RNA polymerase II \\
\hline 6 & 5 & 5 & 7 & NA & 7 & 4 & 4 & 8 & 5 & 5 & oligodendrocyte differentiation \\
\hline 8 & 5 & 8 & 10 & 6 & 13 & 5 & NA & 13 & 10 & 12 & negative regulation of neuron differentiation \\
\hline 10 & 6 & 4 & 6 & 3 & 9 & 3 & NA & 7 & 8 & 5 & somatic stem cell population maintenance \\
\hline 27 & 8 & 10 & NA & NA & 39 & NA & 8 & 25 & 15 & 25 & -log(p-value) \\
\hline 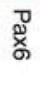 & 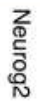 & 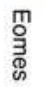 & $\begin{array}{l}z \\
\text { a } \\
\text { co } \\
\stackrel{0}{0}\end{array}$ & $\stackrel{-1}{\underline{\Xi}}$ & 高 & $\begin{array}{l}0 \\
\stackrel{0}{0} \\
\stackrel{0}{0}\end{array}$ & $\begin{array}{l}z \\
\text { ż } \\
\underset{N}{\mathbf{N}}\end{array}$ & $\begin{array}{l}\text { D) } \\
\text { \&्रे }\end{array}$ & 롷 & $\begin{array}{l}\text { के } \\
\stackrel{2}{\underline{n}}\end{array}$ & 12 \\
\hline
\end{tabular}

i

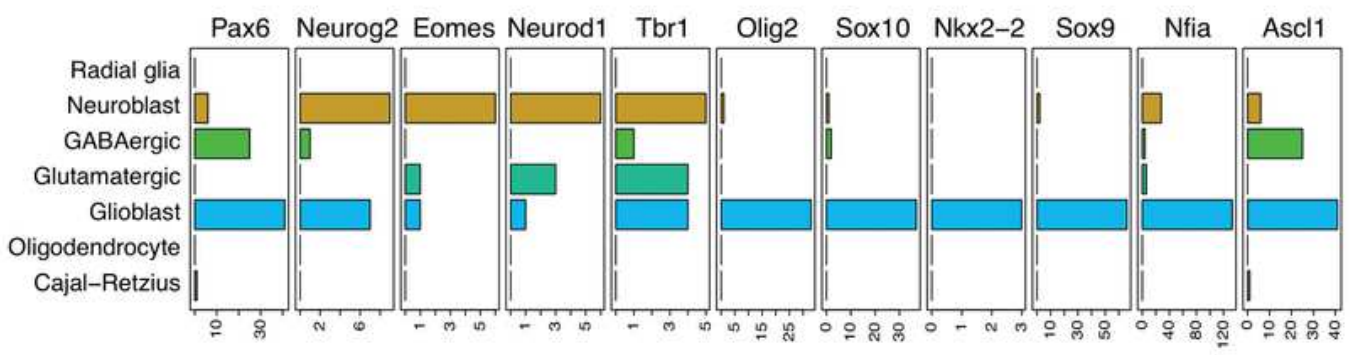

Figure 4

\section{Figure 4}

TRIPOD identified known and novel regulatory relationships during mouse neurogenesis and gliogenesis. 

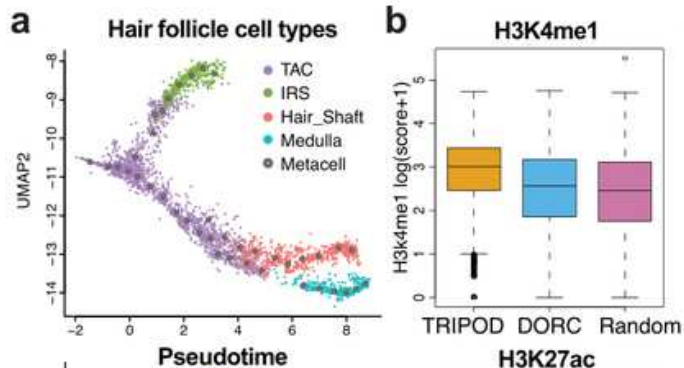

C

TAC superenhancers
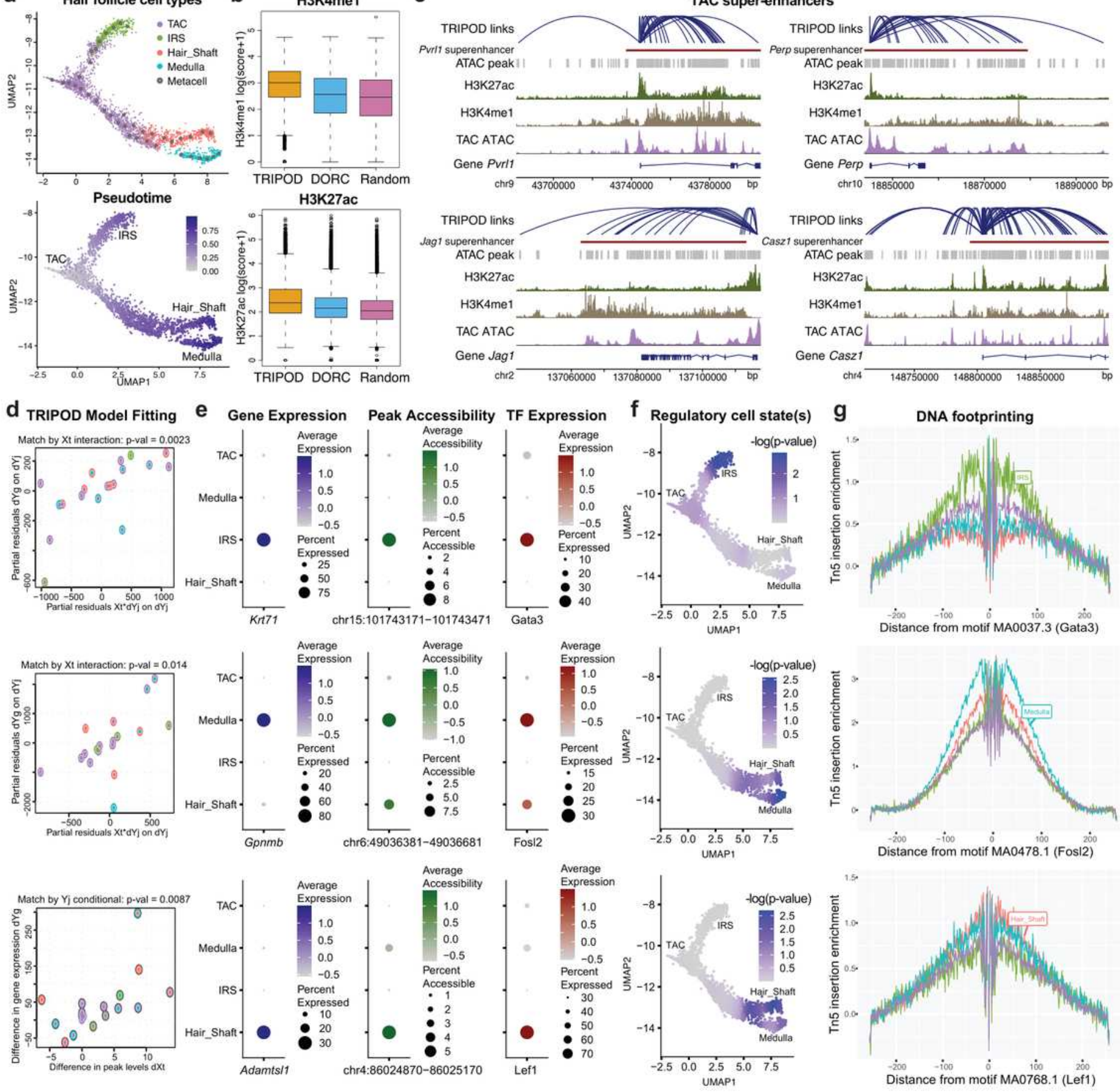

Figure 5

Figure 5

TRIPOD identified regulatory relationships in mouse hair follicles with transient cell states.

\section{Supplementary Files}

This is a list of supplementary files associated with this preprint. Click to download. 
- Table1validationresources.pdf

- TRIPODsupplementsv4.pdf 